PROCEEDINGS OF THE

AMERICAN MATHEMATICAL SOCIETY

Volume 126, Number 10, October 1998, Pages 2849-2853

S 0002-9939(98)04465-7

\title{
A NOTE ON A QUESTION OF J. NEKOVÁŘ AND THE BIRCH AND SWINNERTON-DYER CONJECTURE
}

\author{
KEN ONO
}

(Communicated by David E. Rohrlich)

Abstract. If $D$ is a square-free integer, then let $E(D)$ denote the elliptic curve over $\mathbb{Q}$ given by the equation

$$
E(D): \quad D y^{2}=4 x^{3}-27 .
$$

Let $L(E(D), s)$ denote the Hasse-Weil $L$-function of $E(D)$, and let $L^{*}(E(D), 1)$ denote the 'algebraic part' of the central critical value $L(E(D), 1)$. Using a theorem of Sturm, we verify a congruence conjectured by J. Nekovár. his work, if $S(3, E(D))$ denotes the 3-Selmer group of $E(D)$ and $D \neq 1$ is a square-free integer with $|D| \equiv 1(\bmod 3)$, then we find that

$$
L^{*}(E(D), 1) \not \equiv 0 \quad(\bmod 3) \Longleftrightarrow S(3, E(D))=0 .
$$

If $D \neq 1$ is a square-free integer, then $E(D)$ is the $D$-quadratic twist of the Fermat cubic $x^{3}+y^{3}=1$. J. Nekovár explicitly computed the 3-Selmer ranks of these elliptic curves, and verified the 'mod 3' part of the Birch and Swinnerton-Dyer Conjecture for most of these curves when $|D| \equiv 1(\bmod 3)$ using Waldspurger's theorem on the Shimura correspondence.

If $q:=e^{2 \pi i z}$, and $\eta(z)$ and $\Theta(z)$ denote the usual weight $1 / 2$ modular forms

$$
\eta(z):=q^{1 / 24} \prod_{n=1}^{\infty}\left(1-q^{n}\right) \quad \text { and } \quad \Theta(z):=1+2 \sum_{n=1}^{\infty} q^{n^{2}},
$$

then define $a(n)$ by $\sum_{n=1}^{\infty} a(n) q^{n}:=\eta^{2}(3 z) \eta^{2}(9 z) \in S_{2}\left(\Gamma_{0}(27)\right)$. The Hasse-Weil $L$-function $L(E(D), s)$ is given by

$$
L(E(D), s):=\sum_{n=1}^{\infty} \frac{a(n)\left(\frac{D}{n}\right)}{n^{s}} .
$$

Also let $L^{*}(E(D), 1)$ denote the 'algebraic part' of the critical value $L(E(D), 1)$. In particular, it is given by

$$
L^{*}(E(D), 1):=\frac{L(E(D), 1)}{\Omega(E(D)) \prod_{p} c_{p}},
$$

where $\Omega(E(D))$ is the real period of $E(D)$, and $\prod_{p} c_{p}$ is the 'Tamagawa factor.' In particular if $p$ is an odd prime and $S(p, E(D))$ denotes the $p$-Selmer group of $E(D)$,

Received by the editors March 13, 1997.

1991 Mathematics Subject Classification. Primary 11G40; Secondary 14G10.

Key words and phrases. Elliptic curves, modular forms.

The author is supported by National Science Foundation grants DMS-9304580 and DMS9508976, and NSA grant MSPR-YO12. 
then the Birch and Swinnerton-Dyer Conjecture predicts that

$$
L^{*}(E(D), 1) \not \equiv 0(\bmod p) \Rightarrow S(p, E(D))=0 .
$$

Since the $E(D)$ are curves with complex multiplication by $\mathbb{Q}(\sqrt{-3})$, a theorem of Rubin $[\mathrm{R}]$ implies that

$$
L^{*}(E(D), 1) \not \equiv 0(\bmod p) \Rightarrow S(p, E(D))=0
$$

for primes $p \geq 5$.

If $D$ is square-free and $|D| \equiv 2(\bmod 3)$, then $L(E(D), 1)=L^{*}(E(D), 1)=0$, and so we restrict our attention to those square-free $D$ where $|D| \equiv 1(\bmod 3)$. J. Nekovár computed the 3-Selmer ranks of $E(D)$ and verified (4) when $p=3[\mathrm{~N}$, Cor. 7.5] for all such $D$ except when

$$
0>D \equiv 5 \quad(\bmod 8) \quad \text { and } \quad 1<D \equiv 1 \quad(\bmod 8) .
$$

He explicitly computed 3-Selmer ranks using ideal class groups of suitable quadratic fields, and employed elementary congruences between certain Fourier coefficients of weight $3 / 2$ cusp forms and class numbers.

To prove (4) when $p=3$ for the remaining cases (5), Nekovár noted that it suffices to prove:

Conjecture $([\mathrm{N},(7.1)])$. Define $a_{1}(n)$ and $a_{2}(n)$ by

$$
\begin{aligned}
& \sum_{n=1}^{\infty} a_{1}(n) q^{n}:=\eta(6 z) \eta(18 z) \Theta(3 z), \\
& \sum_{n=1}^{\infty} a_{2}(n) q^{n}:=\eta(6 z) \eta(18 z) \Theta(9 z) .
\end{aligned}
$$

If $h(-n)$ denotes the class number of the quadratic field $\mathbb{Q}(\sqrt{-n})$, then

(i) $\frac{a_{1}(D)}{3} \equiv-h(-D) \quad(\bmod 3) \quad$ if $\quad D \equiv 19 \quad(\bmod 24)$ is square-free,

(ii) $\frac{a_{2}(D)}{3} \equiv-h(-3 D) \quad(\bmod 3) \quad$ if $1<D \equiv 1 \quad(\bmod 24)$ is square-free.

Theorem. Conjecture (7.1) is true.

Corollary. If $D \neq 1$ is a square-free integer for which $|D| \equiv 1(\bmod 3)$, then

$$
L^{*}(E(D), 1) \not \equiv 0(\bmod 3) \Longleftrightarrow S(3, E(D))=0 .
$$

Proof of Corollary. This follows immediately from Theorem 4.6, Proposition 7.1, and Proposition 7.2 in $[\mathrm{N}]$.

Proof of Theorem. Throughout, $k$ denotes a non-negative integer. We begin with a well known fact. If $g(z):=\sum_{n=1}^{\infty} c(n) q^{n} \in M_{k+\frac{1}{2}}\left(\Gamma_{1}(N)\right)$, then

$$
g_{r, t}(z):=\sum_{n \equiv r(\bmod t)} c(n) q^{n} \in M_{k+\frac{1}{2}}\left(\Gamma_{1}\left(\frac{N t^{2}}{\operatorname{gcd}(r, t)}\right)\right) .
$$

We recall a special case of a theorem of Sturm [S]. Suppose that $h(z):=$ $\sum_{n=0}^{\infty} d(n) q^{n} \in M_{k}\left(\Gamma_{1}(M)\right)$ has integer Fourier coefficients. Sturm proved that

$$
h(z) \equiv 0(\bmod S) \Longleftrightarrow \operatorname{Ord}_{S}(h(z))>\frac{k}{12} M^{2} \prod_{p \mid M}\left(1-\frac{1}{p^{2}}\right),
$$


where

$$
\left.\operatorname{Ord}_{S}(h(z)):=\min _{n}(d(n)) \not \equiv 0 \quad(\bmod S)\right) .
$$

If $i(z):=\sum_{n=0}^{\infty} e(n) q^{n} \in M_{k+\frac{1}{2}}\left(\Gamma_{1}(M)\right)$ has integer coefficients, then by applying (7) to $i(z) \Theta(z)$ we find that

$$
i(z) \equiv 0(\bmod S) \Longleftrightarrow \operatorname{Ord}_{S}(i(z))>\frac{k+1}{12} M^{2} \prod_{p \mid M}\left(1-\frac{1}{p^{2}}\right) .
$$

Case $(i)$. The form $\sum_{n=1}^{\infty} a_{1}(n) q^{n}$ is in $S_{\frac{3}{2}}\left(\Gamma_{0}(108), \chi_{0}\right)$, where $\chi_{0}$ is the trivial character, and has the property that $a_{1}(n)=0$ if $n \not \equiv 1(\bmod 3)$. Therefore by $(6)$ we find that

$$
\begin{aligned}
f_{1}(z) & :=\sum_{n \equiv 3(\bmod 8)} a_{1}(n) q^{n} \\
& =\sum_{n \equiv 19} a_{1}(n) q^{n}=-3 q^{19}+6 q^{43}-\cdots \in S_{\frac{3}{2}}\left(\Gamma_{1}\left(108 \cdot 8^{2}\right)\right) .
\end{aligned}
$$

Similarly if $\Theta^{3}(z):=\sum_{n=0}^{\infty} r_{1}(n) q^{n} \in M_{\frac{3}{2}}(4)$, then by (6)

$$
\begin{aligned}
t_{1}(z) & :=\sum_{n \equiv 19(\bmod 24)} r_{1}(n) q^{n} \\
& \left.=24 q^{19}+24 q^{43}+24 q^{67}+48 q^{91}+\cdots \in M_{\frac{3}{2}}\left(4 \cdot 24^{2}\right)\right) .
\end{aligned}
$$

It is easy to verify that $f_{1}(z) \equiv 0(\bmod 3)$ and $t_{1}(z) \equiv 0(\bmod 24)$, and with these observations define $i_{1}(z)$ by

$$
i_{1}(z):=\frac{f_{1}(z)}{3}+\frac{t_{1}(z)}{24}=3 q^{43}+3 q^{91}+6 q^{139}+\cdots \in M_{\frac{3}{2}}\left(\Gamma_{1}(6912)\right) .
$$

A computation verified the congruence $i_{1}(z) \equiv 0(\bmod 3)$ for the first $5,400,000$ terms, and so by (8)

$$
\frac{a_{1}(n)}{3} \equiv-\frac{r_{1}(n)}{24} \quad(\bmod 3)
$$

for every integer $n \equiv 19(\bmod 24)$. Conjecture $7.1(i)$ follows immediately by Gauss' theorem that if $n \equiv 19(\bmod 24)$ is square-free, then $r_{1}(n)=24 h(-n)$ (see $[\mathrm{J}])$.

Case (ii). The form $\sum_{n=1}^{\infty} a_{2}(n) q^{n} \in S_{\frac{3}{2}}\left(\Gamma_{0}(108), \chi_{-3}\right)$ has the property that $a_{2}(n)$ $=0$ if $n \not \equiv 1(\bmod 3)$. Therefore by $(6)$ it turns out that

$$
\begin{aligned}
f_{2}(z) & :=\sum_{n \equiv 1(\bmod 8)} a_{2}(n) q^{n} \\
& =\sum_{n \equiv 1} a_{2}(n) q^{n}=q+q^{25}-2 q^{49}-\cdots \in S_{\frac{3}{2}}\left(\Gamma_{1}\left(108 \cdot 8^{2}\right)\right) .
\end{aligned}
$$

Similarly if $\sum_{n=0}^{\infty} r_{2}(n) q^{n}:=\Theta^{2}(z) \Theta(3 z) \in M_{\frac{3}{2}}\left(\Gamma_{1}(12)\right)$, then by $(6)$

$$
\begin{aligned}
t_{2}(z) & :=\sum_{n \equiv 1(\bmod 24)} r_{2}(n) q^{n} \\
& =4 q+28 q^{25}+28 q^{49}+48 q^{73}+\cdots \in M_{\frac{3}{2}}\left(\Gamma_{1}\left(12 \cdot 24^{2}\right)\right) .
\end{aligned}
$$


It is easy to see that $t_{2}(z) \equiv 0(\bmod 4)$, and so the modular form

$$
f_{2}(z)+\frac{t_{2}(z)}{4}=2 q+8 q^{25}+5 q^{49}+9 q^{73}+9 q^{97}+\cdots \in M_{\frac{3}{2}}\left(\Gamma_{1}\left(12 \cdot 24^{2}\right),\right.
$$

and modulo 9 its first few terms are

$$
f_{2}(z)+\frac{t_{2}(z)}{4} \equiv 2 q+8 q^{25}+5 q^{49}+5 q^{121}+\cdots \quad(\bmod 9) .
$$

Although it is unnecessary, we recall the following eta-function identity:

$$
\frac{\eta^{5}(6 z)}{\eta^{2}(3 z)}=\sum_{1 \leq n \equiv 1,2(\bmod 6)} n q^{n^{2}}-\sum_{0<n \equiv 4,5(\bmod 6)} n q^{n^{2}} \in S_{\frac{3}{2}}\left(\Gamma_{1}(144)\right) .
$$

By (6) it is easy to see that

$$
j_{2}(z):=\sum_{1 \leq n \equiv 1(\bmod 6)} n q^{n^{2}}-\sum_{0<n \equiv 5(\bmod 6)} n q^{n^{2}} \in S_{\frac{3}{2}}\left(\Gamma_{1}(144 \cdot 4)\right) .
$$

Define the form $i_{2}(z) \in M_{\frac{3}{2}}\left(\Gamma_{1}(6912)\right)$ by

$$
i_{2}(z):=f_{2}(z)+\frac{t_{2}(z)}{4}-2 j_{2}(z)=18 q^{25}-9 q^{49}+9 q^{73}+9 q^{97}+\cdots .
$$

After computing the first 5,400,000 terms, by (8) we find that $i_{2}(z) \equiv 0(\bmod 9)$. In particular if $1<n \equiv 1(\bmod 24)$ is square-free, then

$$
a_{2}(n)+\frac{r_{2}(n)}{4} \equiv 0 \quad(\bmod 9) .
$$

Since $r_{2}(n)=\#\left\{x^{2}+y^{2}+3 z^{2}=n \mid x, y, z \in \mathbb{Z}\right\}$, and the ternary form $x^{2}+y^{2}+3 z^{2}$ is in a genus with a single class, by [J, Th. 86] it turns out that $r_{2}(n)=12 h(-3 n)$ if $1<n \equiv 1(\bmod 24)$ is square-free. Therefore for such $n$ it is easy to see by $(9)$ that

$$
\frac{a_{2}(n)}{3} \equiv-h(-3 n) \quad(\bmod 3)
$$

Remark. Using a theorem of Davenport and Heilbronn, as refined by Horie and Nakagawa (see $[\mathrm{D}-\mathrm{H}],[\mathrm{H}-\mathrm{N}]$ ), it is easy to deduce that

$$
\liminf _{x \rightarrow \infty} \frac{\#\{|D|<x \mid \text { square-free }|D| \equiv 1(\bmod 3), \text { with } S(3, E(D))=0\}}{\#\{|D|<x \mid \text { square-free }|D| \equiv 1(\bmod 3)\}} \geq \frac{1}{2} .
$$

In particular, for such $D$ the curves $E(D)$ have rank zero at least half the time. These ideas have been employed by James [Ja], Horie and Nakagawa [H-N], and Wong $[\mathrm{W}]$ to deduce that a positive proportion of certain families of twists of fixed elliptic curves have rank zero.

\section{ACKNOWLEDGEMENTS}

The author is indebted to W. Galway whose computations verified the conjectured congruences. 


\section{REFERENCES}

[D-H] H. Davenport and H. Heilbronn, On the density of discriminants of cubic fields. II., Proc. Roy. Soc. London, Ser. A. 322 (1971), 405-420. MR 58:10816

[Ja] K. James, L-series with non-zero central critical value, Ph.D. Thesis, Univ. Georgia, 1997.

$[\mathrm{J}] \quad$ B. Jones, The arithmetic theory of of quadratic forms, Math. Assoc. Amer., 1950. MR 12:244a

[H-N] K. Horie and J. Nakagawa, Elliptic curves with no rational points, Proc. Amer. Math. Soc. 104 (1988), 20-24. MR 89k:11113

[N] J. Nekovár, Class numbers of quadratic fields and Shimura's correspondence, Math. Ann. 287 (1990), 577-594. MR 91k:11051

[R] K. Rubin, Tate-Shafarevich groups and L-functions of elliptic curves with complex multiplication, Invent. Math. 89 (1987), 527-559. MR 89a:11065

[S] J. Sturm, On the congruence of modular forms, Number Theory (New York, 1984-1985) Ed. D. C. Chudnovsky, G. V. Chudnovsky, M. B. Nathanson, Springer Lect. Notes. Math. 1240 (1984), 275-280. MR 88h:11031

[W] S. Wong, Rank zero twists of elliptic curves, preprint, Brown University.

School of Mathematics, Institute for Advanced Study, Princeton, New Jersey 08540

E-mail address: ono@math.ias.edu

Department of Mathematics, Penn State University, University Park, Pennsylvania 16802

E-mail address: ono@math.psu.edu 